\title{
Hydrolysis of fat by human gastric juice
}

\author{
S. BANK, L. H. KRUT, I. N. MARKS, B. BRONTE-STEWART, \\ AND P. J. LE R. UYS \\ From the Gastrointestinal Service, Groote Schuur Hospital, \\ and the Department of Medicine, University of Cape Town, Observatory, \\ Cape, South Africa
}

EDITORIAL SYNOPSIS It is currently thought that the lipolytic activity of gastric juice is not important. These studies show that human gastric juice produces partial hydrolysis of a triglyceride emulsion and may make a significant initial contribution to the process of fat absorption.

It is established that initial degradation of some dietary foodstuffs in the stomach facilitates subsequent digestion by pancreatic and intestinal enzymes. Salivary amylase coupled with acid hydrolysis initiates the splitting of starch and dextrin to dissacharides, and pepsin- $\mathrm{HCl}$ and pepsins occurring in abnormal conditions (Taylor, 1959) convert dietary protein to proteoses and peptones (Wright, 1952). The importance of gastric juice in preparing starch and protein for further digestion in a readily assimilable form was appreciated by early gastric physiologists (Lancet, 1960) and has not been questioned. This contrasts with the controversial views regarding the role of gastric juice in fat lipolysis. However, the majority of these gastric studies have been concerned with the problem of whether or not a fat-splitting enzyme exists in the stomach.

As early as 1900 Volhard noted that fatty acids could be liberated from fat emulsions if kept in contact with gastric juice or extracts of gastric mucous membrane. He proposed that a specific gastric lipase was present (Volhard, 1900). Experiments in vivo to confirm this hypothesis were indefinite and confused by the difficulty of excluding the possibility of reflux of duodenal juice (Oppenheimer 1925, 1936; Borgström, Dahlqvist, Lundh, and Sjövall, 1957). Studies on gastric juice obtained from Pavlov pouches in dogs proved the existence of a heat-labile factor which was moderately stable in an acid medium; this factor appeared to be capable of producing lipolysis particularly in specimens of gastric juice of low acidity or where the acid was rapidly neutralized (Hull and Keeton, 1917; Takata, 1921). Shønheyder and Volqvartz (1946) confirmed the presence of a gastric lipase which in their view was active primarily on short-chain fatty acids.
Despite the well-known hydrolytic action of mineral acids on fats, little attention has been paid to the role of gastric $\mathrm{HCl}$ in this regard.

We have recently demonstrated an inverse relationship between the degree of gastric acidity and the postprandial lipaemia after feeding fat, and, in addition, studies in vitro showed that a fat emulsion is partially cleared on incubation with gastric juice. This activity in vitro was attributed to the combined action of acid and of enzyme hydrolysis (Marks, Bank, Krut, and Bronte-Stewart, 1962). In the system employed in vitro many results had to be discarded because of the presence of excessive amounts of mucus which interfered with optical density readings. It was apparent that a more efficient method was required to study the extent of gastric lipolysis as well as the nature of the degradation products.

In addition we have compared hydrolysis by gastric juice of predominantly saturated with predominantly unsaturated fats.

\section{MATERIALS AND METHODS}

GASTRIC JUICE Gastric juice was obtained from 38 fasting subjects. Thirty-three of these were healthy, young, medical students and the remaining five were hospital patients free of gastro-duodenal disease. A radio-opaque nasogastric tube was positioned, under fluoroscopic control, to lie in the most dependent part of the stomach and the gastric contents collected by constant suction and frequent aspiration by syringe to ensure patency of the tube. Saliva was rejected into cartons throughout the period of collection.

The fasting contents were discarded and the basal aspirate then collected under ice for one hour. Aliquots of the latter were immediately taken for the study of lipolytic activity in vitro. The volume of secretion in one hour was measured and the acid concentration (mEq./l.) 
and acid output (mEq./hr.) estimated by titrating with $\mathrm{N} / 10$ sodium hydroxide, using phenolphthalein as indicator for total acid determinations.

Only completely clear gastric juice was used in these experiments. All collections containing a visible amount of bile during any period of the one-hour aspiration were outlawed, and the methylene blue test was used to confirm the absence of bile contamination in the remaining collections.

Aliquots of gastric juice from 14 of the above subjects were used to test the degree of hydrolysis of different substrates.

SUBSTRATES A commercially available safflower seed oil emulsion (Arcofac) ${ }^{1}$ was found to be a suitable substrate with regard to uniformity of particulate size and glyceride composition. The proportion of tri- di- and monoglycerides on repeated analyses showed little variation (Table I). A $1.5 \%$ solution (v/v) emulsified with distilled water on a blender was used.

The other substrates used in the same way, were Lipomul ${ }^{2}$, a cotton-seed oil emulsion, $\mathrm{Ediol}^{3}$, a coconut oil emulsion, and also a mixture of animal fats. The last was made up of rendered down beef tallow, lard, and mutton fat in equal proportions emulsified with water and gum acacia. This gave an emulsion with reasonably small particles but not as small, nor as homogeneous in size as the commercial emulsions. In addition, the emulsion was not as stable as the commercial preparations and tended to separate out in the gastric juice.

GASTRIC LIPOLYSIS Three ml. of gastric juice was added to an equal volume of substrate and incubated in a shaking device at $37^{\circ} \mathrm{C}$. for one hour; this period was found to be optimal under these in vitro conditions. After incubation, $5 \mathrm{ml}$. of methanol was added to the incubating mixture and the lipids extracted with petroleum ether (B.P. $40-60^{\circ} \mathrm{C}$.). The tri-, di-, and mono-glycerides were separated on silicic acid columns by the method of Fillerup and Mead (1953), Mead and Fillerup (1954) as modified by Eastman (1963), and glycerides quantitatively determined by the method of Young and Eastman (1963). The extent of hydrolysis was determined by comparing the proportion of tri-, di-, and mono-glyceride in the substrate after incubation with that found on adding aliquots of the same substrate to the same sample of gastric juice which already contained the extracting solvents.

HYDROLYSIS BY ENZYME The proportion of total hydrolysis accounted for by enzyme activity was determined by comparing the proportion of glycerides split on incubating gastric juice with the Arcofac substrate before and after the gastric juice has been heated in boiling water for five minutes.

\section{RESULTS}

GASTRIC SECRETION The mean acid output and

${ }^{1}$ Arcofac, Armour Pharmaceutical Company, England.

${ }^{2}$ Lipomul, The Upjohn Company, Kalamazoo, Michigan, U.S.A.

${ }^{3}$ Ediol, Schenley Laboratories, Inc. Lawrenceburg, Indiana, U.S.A. mean acid concentration in the 38 subjects sampled was $6.0 \pm 5.1 \mathrm{mEq} . / \mathrm{hr}$. and $71 \pm 31 \mathrm{mEq} . / \mathrm{l}$. of 'total acid' respectively. The $p \mathrm{H}$ of the basal secretion ranged from 1 to 5 in 37 of the 38 subjects. Since pancreatic lipase is completely inactivated at a $p \mathrm{H}$ lower than 6 (Marks, et al., 1962), there was little likelihood of pancreatic lipase contributing to the hydrolysis in these tests. The basal secretion in the remaining subject, however, had a $p \mathrm{H}$ of 6.5 but its inclusion was justified by the absence of both bile and amylase on biochemical testing.

LIPOLYTIC ACTIVITY The lipolytic effect of the basal gastric juice is shown in Table I. There was a highly significant $(p<0.001)$ difference between the mean value for triglyceride, expressed as a percentage of total glycerides in the substrate, and the mean percentage value for triglyceride in the lipid extract after one hour's incubation with gastric juice $(91.3 \%$ and $75.3 \%$ respectively). Gastric juice was thus found to produce partial hydrolysis of the trigylceride emulsion to the extent of $16.0 \pm 9.4 \%$ with a range of 0.8 to $39 \%$ under the conditions of the experiment (Table I). Hydrolysis was further evidenced by the significant increase in the proportion of diglycerides $(\mathrm{p}<0.001)$ and monoglycerides $(\mathrm{p}<0.001)$ although the last change was quantitatively very small. Liberated fatty acids were not estimated.

\section{TABLE I}

PROPORTIONS OF TRI-, DI-, AND MONOGLYCERIDES BEFORE AND AFTER INCUBATION OF THE SUBSTRATE WITH GASTRIC JUICE ${ }^{1}$

\begin{tabular}{|c|c|c|c|c|c|c|}
\hline & Subs & & & After & cubati & \\
\hline & Tri- & $D i-$ & Mono- & Tri- & $D i-$ & Mono- \\
\hline $\begin{array}{l}\text { Mean }(\%) \\
\text { S.D. }\end{array}$ & $\begin{array}{r}91 \cdot 3 \\
2 \cdot 2\end{array}$ & $\begin{array}{l}7 \cdot 7 \\
2 \cdot 2\end{array}$ & $\begin{array}{l}1 \cdot 0 \\
0.8\end{array}$ & $\begin{array}{r}75 \cdot 3 \\
9.9\end{array}$ & $\begin{array}{r}21 \cdot 0 \\
8 \cdot 1\end{array}$ & $\begin{array}{l}3 \cdot 7 \\
3 \cdot 2\end{array}$ \\
\hline
\end{tabular}

${ }^{1}$ The difference in the proportions are all highly significant $(P<0.001)$.

LIPOLYTIC ACTIVITY IN RELATION TO ACID SECRETION The findings of an inverse correlation between acid concentration and the degree of post-prandial lipaemia (Marks et al., 1962) prompted us to examine the relationship between the extent of hydrolysis and degree of gastric acidity. No correlation was found between the percentage splitting and the degree of gastric acidity $(r=-0.05)$. Indeed, the reverse appeared to be the case, i.e., where lipolysis was low, acidity tended to be high and vice versa. From Fig. 1 it can be seen that both the mean acid concentration and mean acid output were, on the average, highest when the gastric lipolytic activity was low whereas low acid secretion was associated with higher lipolytic activity. 


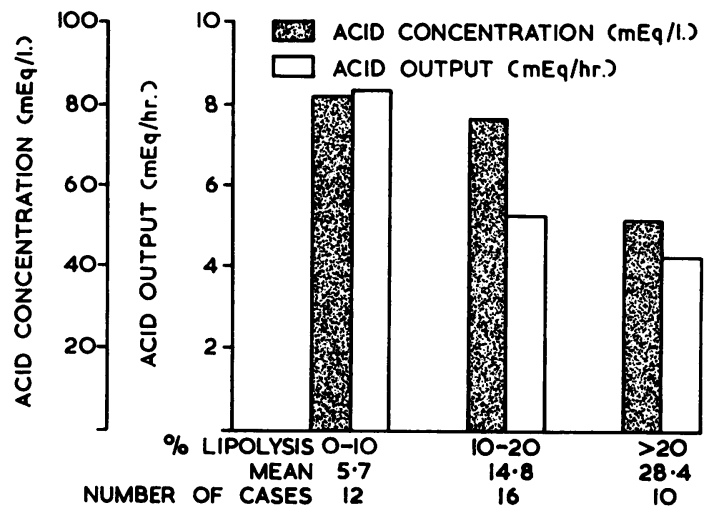

FIG. 1. The relation between the degree of acidity in the gastric juice, shown as a mean, and the extent of lipolysis, expressed as the change in the proportion of triglycerides after incubation.

EFFECT ON LIPOLYTIC ACTIVITY ON HEATING GASTRIC JUICE The relative contribution of acid hydrolysis and presumed enzymatic activity of gastric juice was studied in 12 of the 38 subjects (Table II). This was done by comparing the lipolytic activity of heated and unheated gastric juice. A significant difference $(p<0.01)$ was found in the degree of lipolysis between the heated and unheated samples. Before incubating, the mean proportion of triglyceride in the substrate was $90.1 \%$. The mean proportion of triglycerides after incubation with unheated juice was $65.5 \%$ compared with a mean value of $80.5 \%$ obtained after preliminary heating of the gastric juice. Thus of a mean lipolytic activity in the 12 patients of about $25 \%, 10 \%$ could be accounted for by acid hydrolysis and the remaining $15 \%$ by a heat-labile factor, presumed to be enzymatic in nature. The mean acid output in these 12 subjects was $4.5 \pm 3.4 \mathrm{mEq}$. $/ \mathrm{hr}$. which was not significantly different from the remaining subjects $(p<0 \cdot 2)$.

LIPOLYTIC ACTIVITY ON PREDOMINANTLY SATURATED AND PREDOMINANTLY UNSATURATED FATS It is apparent from Table III that the degree of hydrolysis, when expressed as the percentage change in the proportion of triglycerides after incubation, tends to be greater for the predominantly unsaturated fats.
On statistical analysis both Arcofac and Lipomul were hydrolysed to a greater degree than the animal fat $(\mathrm{p}<0.02$ and $<0.001$ respectively). Neither Arcofac not Lipomul, however, were split to a significantly greater degree than was Ediol $(p<0.5$ and $<0.2$ respectively). There was no significant difference between the degree of hydrolysis of Ediol and animal fat $(\mathrm{p}<0 \cdot 2)$.

\section{TABLE III}

DEGREE OF HYDROLYSIS, EXPRESSED AS THE CHANGE IN PROPORTION OF TRIGLYCERIDE AFTER INCUBATION, SHOWN AS A MEAN, WITH ONE STANDARD DEVIATION

(S.D.), AND RANGE FOR THE DIFFERENT SUBSTRATES

\begin{tabular}{|c|c|c|c|}
\hline Substrate & & Mean (S.D.) & Range \\
\hline $\begin{array}{l}\text { Arcofac } \\
\text { Lipomul } \\
\text { Ediol } \\
\text { Animal fat }\end{array}$ & 1 & $\begin{array}{r}14.0(11.9) \\
15.8(7.5) \\
10.4(13.1) \\
4.2(5.3)\end{array}$ & $\begin{array}{r}0 \cdot 7-41 \cdot 6 \\
0-24 \cdot 4 \\
0-36 \cdot 5 \\
0-16 \cdot 5\end{array}$ \\
\hline
\end{tabular}

Arrows join values where the difference is significant. ${ }^{1} \mathrm{P}<0.001 \quad{ }^{2} \mathrm{P}<0.02$

The most striking finding in this part of the study was the marked variability in the degree of hydrolysis of the different substrates between different samples of gastric juice. In general, where lipolytic activity was high, the predominantly saturated fats tended to be split to much the same degree as the predominantly unsaturated fats. Where lipolytic activity was low, the predominantly unsaturated fats tended to show greater hydrolysis. The animal fat was often an exception showing, fairly consistently, poor hydrolysis which may have been due to the poor stability and greater particle size of this emulsion. The data would favour the view that the extent of hydrolysis reflects the lipolytic potential of the gastric juice as such rather than the degree of saturation of the substrate.

In this subgroup the trend for the degree of lipolysis to decrease with increasing acidity was also evident and appeared to hold for all substrates.

\section{DISCUSSION}

The current views on fat digestion in the stomach emphasize that the lipolytic activity of gastric juice is not important (Harper, 1961) and that any fat

\section{TABLE II}

HYDROLYSIS BY UNHEATED AND HEATED GASTRIC JUICE COMPARED ${ }^{1}$

Mean $(\%)(n=12)$

S.D.

\begin{tabular}{cll}
\multicolumn{2}{l}{ Substrate } & \\
\hline Tri- & Di- & Mono- \\
\hline $90 \cdot 1$ & $8 \cdot 1$ & $1 \cdot 8$ \\
$2 \cdot 5$ & $2 \cdot 0$ & 0.8
\end{tabular}

\begin{tabular}{crl}
\multicolumn{3}{l}{ Unheated Gastric Juice } \\
\hline Tri- & Di- & Mono- \\
\hline 65.5 & 27.0 & 7.5 \\
9.5 & 9.7 & 4.8
\end{tabular}

\begin{tabular}{rrl}
\multicolumn{3}{l}{ Heated Gastric Juice } \\
\hline Tri- & Di- & Mono- \\
\hline 80.5 & $15 \cdot 2$ & $4 \cdot 3$ \\
7.7 & 6.1 & $5 \cdot 2$
\end{tabular}

${ }^{1}$ The proportions of tri-, di-, and mono-glycerides on incubating the substrate with heated gastric juice are all highly significant (P<0.001), differing from that obtained with unheated gastric juice. 
splitting is probably due to regurgitated pancreatic lipase (Bockus, 1963). The results of the present experiments, however, clearly demonstrate that human gastric juice is able to produce partial hydrolysis of a triglyceride emulsion. It has also been demonstrated that lipolysis by gastric juice represents the effect both of acid and of a heat-labile factor, presumably a gastric lipase. That this enzyme is at least to some extent stable in an acid medium is reflected by the considerable lipolytic effect that could be attributed to enzyme action in samples of gastric juice with high acidity.

EXTENT OF GASTRIC LIPOLYSIS These findings suggest that the major effect of fat digestion in the stomach matches protein and carbohydrate hydrolysis where the hydrolytic action is incomplete (Lancet, 1960) and results in the liberation of initial breakdown products. There is some evidence that the nature of the fat substrate may influence the degree of hydrolysis. Herting and Ames (1955) in experiments in rats in vivo found high fatty acid levels in the gastric contents after feeding saturated fats but not after feeding predominantly unsaturated fats. Their findings are, however, subject to the criticism that they were measuring only gastric residue and they had no way of estimating what products might have left the stomach or to what extent they were measuring regurgitated duodenal contents. Our studies, comparing the lipolytic activity of gastric juice on predominantly saturated with predominantly unsaturated fats (Table III), indicate that the extent of hydrolysis seems to be dependent on the capacity of the sample of gastric juice to hydrolyse fat rather than on the type of triglyceride used as substrate.

Three factors require consideration in assessing the amount of lipolysis which could occur in the stomach:-

1 Length of time fat is retained in the stomach after ingestion Although McLure, Reynolds, and Schwartz (1920) showed that gastric emptying began within three to 10 minutes after a meal, complete emptying may be delayed for four to six hours. After a large meal the initial rapid efflux from the stomach is followed by a 'slowly diminishing trickle' which might be related to further digestion (Hunt and McDonald, 1954). Reduction in the coordinated propulsive activity of the antrum and subsequent slowing of gastric emptying after a fatty meal may represent a physiological mechanism aimed at promoting maximal hydrolysis in the stomach.

$2 \mathrm{pH}$ of the gastric juice We have found that lipolysis will occur at a wide range of $p \mathrm{H}$ but there is a trend for the extent of lipolysis to increase with decreasing acidity. This is in accord with findings of previous workers (Hull and Keeton, 1917; Takata,
1921; Shønheyder and Volqvarts, 1946), who reported the optimum $p \mathrm{H}$ for gastric lipase to be $4-5$, or higher. It is tempting to speculate that inhibition of gastric acid secretion via the enterogastrone mechanism following the entry of fat into the duodenum in sufficiently high concentration might be a physiological means for augmenting the hydrolysis of fat by increased lipase action.

3 Particle size The rapidity and extent of lipolysis depends on the size of the particles dispersed in the emulsion; the smaller the particle the larger will be the total surface area exposed to hydrolytic factors. While we have shown that fat emulsions can undergo considerable partial hydrolysis, it may be argued that much of normally ingested triglyceride is in solid or semi-solid form. It is likely, however, that the combined effect of mastication, gastric peristalsis, body temperature, and acid breakdown into smaller units contributes to the formation of particles suitable for effective hydrolysis.

POSSIBLE PHYSIOLOGICAL ROLE OF GASTRIC LIPOLYSIS The relatively minor degree of hydrolysis in the stomach would seem to contribute little to fat digestion on a quantitative basis when compared with the potent fat-splitting potential of pancreatic lipase. This technique, however, probably does not reflect the possible extent of gastric lipolysis if one considers the time fat is in contact with gastric secretion in vivo. It is, however, established that lower glycerides and free fatty acids together with bile salts provide an efficient emulsifying system. It is thus conceivable that partial hydrolysis by gastric juice, in preparing the fat substrate in a form optimal for the action of pancreatic lipase, might determine the degree of ultimate hydrolysis lower down in the gastrointestinal tract.

The loss of initial gastric lipolysis secondary to reduced acidity and loss of reservoir and triturating function of the stomach may have a bearing on the defective fat absorption which is commonly encountered after partial gastrectomy and almost universally after total gastric resection.

Whether there is any relation between the extent of intestinal lipolysis and the degree of post-prandial lipaemia after a fat meal in subjects free from overt gastrointestinal disease is not known. Our previous studies showed an inverse correlation between the degree of post-prandial lipaemia and gastric acid secretion in that the high acid secretors had the lower glyceridaemia and vice versa. It thus appeared that gastric lipolytic potential might be related to the degree of post-prandial lipaemia. This finding would appear to be at variance with the results in the present study where the high acid juices have lower lipolytic activity (Fig. 1). In our previous study, 
however, the fat was placed in the stomach via a gastric tube together with the pooled gastric secretions obtained after maximal histamine stimulation. In this highly acid juice the major lipolytic factor might have been acid and not enzyme.

\section{SUMMARY}

Basal gastric juice from 38 subjects was examined for lipolytic activity on incubation with a fat substrate in vitro. The degree of lipolysis could be attributed to acid hydrolysis and to the action of a heat-labile factor, presumably a gastric lipase. Although enzyme lipolysis occurred at wide ranges of $p \mathrm{H}$, the effect was greater the lower the acidity.

While the extent of gastric lipolysis in vivo cannot be deduced from this study the data favour the view that to a considerable extent fat may be split in the stomach.

The degree of lipolysis of predominantly saturated fats tended to be lower than that of predominantly unsaturated fats. In general, however, the degree of hydrolysis appears to be dependent on the lipolytic activity of the gastric juice rather than on the degree of saturation of the fat substrate.

The theoretical implications of initial partial hydrolysis by gastric juice are discussed.

This study was supported in part by research grant HE-O3316 from the National Institute of Health, Public Health Service, U.S.A., the South African Council for Scientific and Industrial Research, the Ben May Gastroenterology Research Fund, and the C.L. Herman Bequest to the University of Cape Town.

\section{REFERENCES}

Bockus, H. L. (1963). Gastroenterology, 2nd ed., vol. I, p. 277. Saunders, Philadelphia and London.

Borgström, B., Dahlqvist, A., Lundh, G., and Sjövall, J. (1957). Studies of intestinal digestion and absorption in the human. J. clin. Invest., 36, 1521-1536.

Eastman, R. (1963). A micromethod for the quantitative determination of mono-, di-, and tri-glycerides in blood serum. S. Afri.J. Lab. clin. Med., 8, 69-72.

Fillerup, D. L., and Mead, J. F. (1953). Chromatographic separation of the plasma lipids. Proc. soc. exp. Biol. N.Y., 83, 574-577.

Harper, H. A. (1961). Review of Physiol. Chemistry, p. 146. Lange Med. Publications, California.

Herting, D. C., and Ames, S. R. (1955). On the gastric lipolysis of fat. Arch. biochem., 55, 295-297.

Hull, M., and Keeton, R. W. (1917). The existence of a gastric lipase. J. biol. Chem., 32, 127-140.

Hunt, J. N., and MacDonald, I. (1954). The influence of volume on gastric emptying. J. Physiol. (Lond.), 126, 459-474.

Lancet (1960). Editorial. The role of the stomach in digestion. 1, 322-324.

Marks, I. N., Bank, S., Krut, L. H., and Bronte-Stewart, B. (1962). Gastric secretion and alimentary lipaemia in ischaemic heartdisease. Lancet, 21 1068-1072.

McClure, C. W., Reynolds, L., and Schwartz, C. O. (1920). On the behaviour of the pyloric sphincter in normal man. Arch. intern. Med., 26, 410-423.

Mead, J. F., and Fillerup, D. L. (1954). Plasma lipids in fat deficiency. Proc. Soc. exp. Biol. N.Y., 86, 449-451.

Oppenheimer, C. (1925). Die Fermente und ihre Wirkungen, 5 ed. vol. I, p. 487. Thieme, Leipzig. Cited by Shønheyder and Volqvartz (1946).

- (1936). Die Fermente und ihre Wirkungen, suppl., vol. I, p. 60. Junk, The Hague. Cited by Shønheyder and Volqvartz (1946).

Shønheyder, F., and Volqvartz, K. (1946). The gastric lipase in man. Acta physiol. scand., 11, 349-360.

Takata, M. (1921). Studies in the gastric juice. II. On the action and properties of the gastric lipase (Part I). Tohoku J. exp. Med., 2, 209-257.

Taylor, W. H. (1959). Studies on gastric proteolysis. I. The proteolytic activity of human gastric juice and pig and calf gastric mucosal extracts below $p \mathrm{H}$ 5. Biochem. J., 71, 73-83.

Volhard, F. (1900). Ueber Resorption und Fettspaltung im Magen. Münch. med. Wschr., 47, 141-146.

Wright, S. (1952). Applied Physiology, 9th ed., p. 878. Oxford University Press, London.

Young, G., and Eastman, R. (1963). A micromethod for the determination of serum triglycerides. S. Afr. J. Lab. clin. Med., 9, 28-31. 